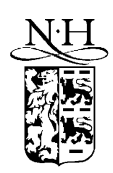

\title{
Anholonomic frames, generalized Killing equations, and anisotropic Taub-NUT spinning spaces
}

\author{
Sergiu I. Vacaru ${ }^{\mathrm{a}, \mathrm{b}}$, Ovidiu Tintareanu-Mircea ${ }^{\mathrm{c}}$ \\ a Physics Department, CSU Fresno, Fresno, CA 93740-8031, USA \\ ${ }^{\mathrm{b}}$ Centro Multidisciplinar de Astrofisica-CENTRA, Departamento de Fisica, Instituto Superior Tecnico, \\ Av. Rovisco Pais 1, Lisboa, 1049-001, Portugal \\ ${ }^{\mathrm{c}}$ Institute of Space Sciences, P.O. Box MG-23, RO 76911, Magurele, Bucharest, Romania
}

Received 30 July 2001; accepted 16 January 2002

\begin{abstract}
By using anholonomic frames in (pseudo)-Riemannian spaces we define anisotropic extensions of Euclidean Taub-NUT spaces. With respect to coordinate frames such spaces are described by offdiagonal metrics which could be diagonalized by corresponding anholonomic transforms. We define the conditions when the 5D vacuum Einstein equations have as solutions anisotropic Taub-NUT spaces. The generalized Killing equations for the configuration space of anisotropically spinning particles (anisotropic spinning space) are analyzed. Simple solutions of the homogeneous part of these equations are expressed in terms of some anisotropically modified Killing-Yano tensors. The general results are applied to the case of the four-dimensional locally anisotropic Taub-NUT manifold with Euclidean signature. We emphasize that all constructions are for (pseudo)-Riemannian spaces defined by vacuum solutions, with generic anisotropy, of 5D Einstein equations, the solutions being generated by applying the moving frame method. @ 2002 Elsevier Science B.V. All rights reserved.
\end{abstract}

PACS: 12.10.-g; 03.50.-z

\section{Introduction}

Much attention has been paid to off-diagonal metrics in higher-dimensional gravity beginning the Salam, Strathee and Perracci work [1] which showed that including offdiagonal components in higher-dimensional metrics is equivalent to including $U(1), S U(2)$

E-mail addresses: vacaru@ fisica.ist.utl.pt, sergiu_vacaru@yahoo.com (S.I. Vacaru), ovidiu@venus.nipne.ro (O. Tintareanu-Mircea). 
and $S U(3)$ gauge fields. The approach was developed by construction of various locally isotropic solutions of vacuum 5D Einstein equations describing 4D wormholes and/or flux tube gravitational-electromagnetic configurations (see Ref. [2]).

Recently, the off-diagonal metrics were considered in a new fashion by applying the method of anholonomic frames with associated nonlinear connections [3-5] which allowed to construct new classes of solutions of Einstein's equations in three (3D), four (4D) and five (5D) dimensions which had generic local anisotropy, e.g., static black hole and cosmological solutions with ellipsoidal or toroidal symmetry, various soliton-dilaton 2D and 3D configurations in 4D gravity, and wormhole and flux tubes with anisotropic polarizations and/or running constants with different extensions to backgrounds of rotation ellipsoids, elliptic cylinders, bipolar and toroidal symmetry and anisotropy.

Another class of 4D metrics induced from 5D Kaluza-Klein theory is connected with the Euclidean Taub-NUT metric which is involved in many modern studies of physics, for instance, in definition of the gravitational analogue of the Yang-Mills instantons [6] and of Kaluza-Klein monopole [7] related with geodesic geometry of higher-dimensional (pseudo)-Riemannian spaces [8] (see a recent review and original results in [9]).

The construction of monopole and instanton solutions, with deformed symmetries, in modern string theory, extra-dimensional gravity and quantum chromodynamics is of fundamental importance in understanding these theories (especially their non-perturbative aspects). Such solutions are difficult to find, and the solutions which are known usually have a high degree of symmetry. In this work we apply the method of anholonomic frames to construct the general form anholonomically constrained Taub-NUT metrics in 5D Kaluza-Klein theory. These solutions have local anisotropy which would make their study using holonomic frames difficult. This helps to demonstrate the usefulness of the anholonomic frames method in studying anisotropic solutions. Most physical situations do not possess a high degree of symmetry, and so the anholonomic frames method provides a useful mathematical framework for studying these less symmetric configurations.

We emphasize that the anholonomic moving frame method works effectively in construction of anisotropic mass hierarchies with running of constants in modern brane physics [10] (on new directions in extra-dimensional gravity see Ref. [11]). This allows us to approach a task of of primordial importance of definition of non-perturbative models and finding of exact solutions in higher-dimensional field theory describing anisotropic monopole/instanton configurations with running constants.

The metrics considered for both wormhole and Taub-NUT geometry and physics could be given by $5 \mathrm{D}$ line elements with $3 \mathrm{D}$ spherical coordinates $(r, \theta, \varphi)$,

$$
\begin{aligned}
d s_{5}^{2}= & -e^{2 v(r)} d t^{2}+d s_{4}^{2}, \\
d s_{4}^{2}= & V^{-1}(r)\left[d r^{2}+a(r)\left(d \theta^{2}+\sin ^{2} \theta d \varphi^{2}\right)\right] \\
& +16 m_{0}^{2} V(r) r_{0}^{2} e^{2 \psi(r)-2 v(r)}\left[d \chi+\omega(r) d t+n_{0} \cos \theta d \varphi\right]^{2},
\end{aligned}
$$

where the metric coefficients and constants $m_{0}, r_{0}, n_{0}$ have to be correspondingly parametrized in order to select two particular cases: 
1. We must put $a(r)=r^{2}, r_{0}^{2} e^{2 \psi(r)-2 v(r)}=1, n_{0}=1, \omega(r)=0, v(r)=0, m_{0}=$ const,

$$
V(r)=\left(1+\frac{4 m_{0}}{r}\right)^{-1}
$$

and to impose on the fifth coordinate the condition $0 \leqslant \chi<4 \pi, 4 m_{0}(\chi+\varphi)=-x^{5}$, if we want to obtain the Taub-NUT metric connected with the gauge field $\vec{A}$ of a monopole

$$
\begin{aligned}
& A_{r}=A_{\varphi}=0, \quad A_{\theta}=4 m_{0}(1-\cos \theta), \\
& \vec{B}=\operatorname{rot} \vec{A}=\frac{4 m_{0} \vec{r}}{r^{3}},
\end{aligned}
$$

where $\vec{r}$ denotes a three-vector $\vec{r}=(r, \theta, \varphi)$; the so-called NUT singularity is absent if $x^{5}$ is periodic with period $16 \pi m_{0}[12]$.

2. The wormhole/flux tube metrics [2] are parametrized if we put $V(r)=1$ and $16 m_{0}^{2}=1$ for $r \in\left\{-R_{0},+R_{0}\right\}\left(R_{0} \leqslant \infty\right), r_{0}=$ const; all functions $v(r), \psi(r)$ and $a(r)$ are taken to be even functions of $r$ satisfying the conditions $v^{\prime}(0)=\psi^{\prime}(0)=a^{\prime}(0)=0$. The coefficient $\omega(r)$ is treated as the $t$-component of the electromagnetic potential and $n_{0} \cos \theta$ is the $\varphi$-component. These electromagnetic potentials lead to radial KaluzaKlein 'electrical' $E_{\mathrm{KK}}$ and 'magnetic' $H_{\mathrm{KK}}$ fields:

$$
E_{\mathrm{KK}}=r_{0} \omega^{\prime} e^{3 \psi-4 v}=q_{0} / a(r)
$$

with the 'electric' charge $q_{0}=r_{0} \omega^{\prime}(0)$ and

$$
H_{\mathrm{KK}}=Q_{0} / a(r)
$$

with 'magnetic' charge $Q_{0}=n_{0} r_{0}$.

The solution in [13] satisfied the boundary conditions $a(0)=1, \psi(0)=v(0)=0$, where it was proved that the free parameters of the metric are varied there are five classes of wormhole/flux tube solutions.

We note that the metric (1) defines solutions of vacuum Einstein equations only for particular parametrizations of type 1 or 2 ; it is not a vacuum solution for arbitrary values of coefficients.

The main results of works [4,5] were obtained by applying the anholonomic frame method which allowed to construct off-diagonal metrics describing wormhole/flux tube configurations with anisotropic varying on the coordinates $\chi$ or $\varphi$ given by some higherdimensional renormalizations of the constants $r_{0} \rightarrow \hat{r}_{0}(\ldots, \chi)$ (or, inversely, $\rightarrow \hat{r}_{0}(\ldots, \varphi)$ ) and/or $n \rightarrow \hat{n}(\ldots, \chi)$ (or, inversely, $\rightarrow \hat{n}(\ldots, \varphi)$ ).

The purpose of this paper is to construct Taub-NUT like metrics with anisotropic variations of the constant $m_{0}$, when $m_{0} \rightarrow m(\ldots, \chi)$, or $m_{0} \rightarrow m(\ldots, \varphi)$. We note that such anisotropic metrics are given by off-diagonal coefficients which define solutions of the 5D vacuum Einstein equations and generalize the constructions from $[12,14]$ to Taub-NUT locally anisotropic gravitational instantons embedded into anisotropic variants of Kaluza-Klein monopoles (the first anisotropic instanton solutions were proposed in 
Ref. [15] for the so-called generalized Finsler-Kaluza-Klein spaces and locally anisotropic gauge gravity, here we note that in this paper we shall not concern topics relating generalized Lagrange and Finsler (super)-spaces [3]); by using anholonomic frames we can model anisotropic instanton configurations in usual Riemannian spaces. The anisotropic metrics are defined as (pseudo)-Riemannian ones which admit a diagonalization with respect to some anholonomic frame bases with associated nonlinear connection structures. Such spacetimes, provided with metrics with generic anisotropy and anholonomic frame structure, are called as anisotropic spaces-time.

Let us introduce a new 5th coordinate

$$
\varsigma=\chi-\int \mu(\theta, \varphi)^{-1} d \xi(\theta, \varphi)
$$

for which $d \chi+n_{0} \cos \theta d \varphi=d \varsigma+n_{0} \cos \theta d \theta$ and

$$
\frac{\partial \xi}{\partial \varphi}=\mu n_{0} \cos \theta, \quad \frac{\partial \xi}{\partial \theta}=-\mu n_{0} \cos \theta,
$$

if the factor $\mu(\theta, \varphi)$ is taken, for instance,

$$
\mu(\theta, \varphi)=|\cos \theta|^{-1} \exp (\theta-\varphi), \quad \text { for } \cos \theta \neq 0 .
$$

With respect to the new extra-dimensional coordinate $\varsigma$ the component $A_{\varphi}$ of the electromagnetic potential is removed into the component $A_{\theta}$; this will allow us to treat the coordinates $(t, r, \theta)$ as holonomic coordinates but $(\varphi, \varsigma)$ as anholonomic ones.

For our further considerations it is convenient to use a conformally transformed (multiplied on the factor $V(r)$ ) Taub-NUT metric with the 5th coordinate $\varsigma$

$$
\begin{aligned}
d s^{2}= & -d t^{2}+d r^{2}+r^{2}\left(d \theta^{2}+\sin ^{2} \theta d \varphi^{2}\right) \\
& +16 m_{0}^{2} V^{2}(r)[d \varsigma+\cos \theta d \theta]^{2}
\end{aligned}
$$

which will be used for generalizations in order to obtain new solutions of the vacuum Einstein equations, being anisotropic on coordinates $(\theta, \varphi, \varsigma)$. This metric generates a monopole configuration (3).

The paper is organized as follow:

Section 2 outlines the geometry of anholonomic frames with associated nonlinear connections on (pseudo)-Riemannian spaces. The metric ansatz for anisotropic solutions is introduced.

In Section 3 there are analyzed the basic properties of solutions of vacuum Einstein equations with mixed holonomic and anholonomic variables. The method of construction of exact solutions with generic local anisotropy is developed.

In Section 4 we construct three classes of generalized anisotropic Taub-NUT metrics, being solutions of the vacuum Einstein equations, which posses anisotropies of parameter $m$ on angular coordinate $\varphi$, or contains a running constant $m(\varsigma)$ and/or are elliptically polarized on angular coordinate $\theta$.

Section 5 is devoted to a new exact 5D vacuum solution for anisotropic Taub-NUT wormholes obtained as a nonlinear superposition of the running on extra-dimensional coordinate Taub-NUT metric and a background metric describing locally isotropic wormhole/flux tube configurations. 
Section 6 elucidates the problem of definition of integrals of motion for anholonomic spinning of particles in anisotropic spaces. There are introduced Killing, energy and momentum and Runge-Lenz vectors with respect to anholonomic bases with associated nonlinear connection structures defined by anisotropic solutions of vacuum Einstein equations. There are proposed and analyzed the action for anisotropic spinning of particles, defined the Poisson-Dirac brackets on anisotropic spaces. We consider anisotropic Killing equations and discuss the problem of construction their generic solutions and non-generic solutions with anholonomic Killing-Yano tensors.

In Section 7 we approach the problem of definition of Killing-Yano tensors for anisotropic Taub-NUT spinning spaces and construct the corresponding Lie algebra with anisotropic variation of constants.

Finally, in Section 8 some conclusion remarks are presented.

\section{Anholonomic frames and nonlinear connections in Riemannian spaces}

In this section we outline the basic formulas on anholonomic frames with mixed holonomic-anholonomic components (variables) and associated nonlinear connection structures in Riemannian spaces.

\subsection{Metric ansatz}

Let us consider a 5D pseudo-Riemannian spacetime of signature $(-,+,+,+,+)$ and denote the local coordinates

$$
u^{\alpha}=\left(x^{i}, y^{a}\right)=\left(x^{1}=t, x^{2}=r, x^{3}=\theta, y^{4}=s, y^{5}=p\right),
$$

where $(s, p)=(\varsigma, \varphi)$, or, inversely, $(s, p)=(\varphi, \varsigma)$-or more compactly $u=(x, y)$ where the Greek indices are conventionally split into two subsets $x^{i}$ and $y^{a}$ labeled, respectively, by Latin indices of type $i, j, k, \ldots=1,2,3$ and $a, b, \ldots=4,5$. The local coordinate bases, $\partial_{\alpha}=\left(\partial_{i}, \partial_{a}\right)$, and their duals, $d^{\alpha}=\left(d^{i}, d^{a}\right)$, are written, respectively, as

$$
\partial_{\alpha} \equiv \frac{\partial}{d u^{\alpha}}=\left(\partial_{i}=\frac{\partial}{d x^{i}}, \partial_{a}=\frac{\partial}{d y^{a}}\right)
$$

and

$$
d^{\alpha} \equiv d u^{\alpha}=\left(d^{i}=d x^{i}, d^{a}=d y^{a}\right) .
$$

The 5D (pseudo)-Riemannian squared linear interval

$$
d s^{2}=g_{\alpha \beta} d u^{\alpha} d u^{\beta}
$$

is given by the metric coefficients $g_{\alpha \beta}$ (a matrix ansatz defined with respect to the coordinate frame base (9)) in the form

$$
\left[\begin{array}{ccccc}
-1 & 0 & 0 & 0 & 0 \\
0 & g_{2}+w_{2}^{2} h_{4}+n_{2}^{2} h_{5} & w_{2} w_{3} h_{4}+n_{2} n_{3} h_{5} & w_{2} h_{4} & n_{2} h_{5} \\
0 & w_{3} w_{2} h_{4}+n_{2} n_{3} h_{5} & g_{3}+w_{3}^{2} h_{4}+n_{3}^{2} h_{5} & w_{3} h_{4} & n_{3} h_{5} \\
0 & w_{2} h_{4} & w_{3} h_{4} & h_{4} & 0 \\
0 & n_{2} h_{5} & n_{3} h_{5} & 0 & h_{5}
\end{array}\right]
$$


where the coefficients are some necessary smoothly class functions of type:

$$
\begin{aligned}
& g_{2,3}=g_{2,3}(r, \theta)=\exp \left[2 b_{2,3}(r, \theta)\right], \\
& h_{4,5}=h_{4,5}(r, \theta, s)=\exp \left[2 f_{4,5}(r, \theta, s)\right], \\
& w_{2,3}=w_{2,3}(r, \theta, s), \quad n_{2,3}=n_{2,3}(r, \theta, s) ;
\end{aligned}
$$

one considers dependencies of the coefficients of metric on two so-called isotropic variables $(r, \theta)$ and on one anisotropic variable, $y^{4}=s$ (in similar fashions we can alternatively consider dependencies on arbitrary couples of $x$-coordinates completed with one $y$-coordinate, for instance, $(r, \theta)$ and $(r, \theta, p))$.

The metric (10) with coefficients (11) can be equivalently rewritten in the form

$$
\delta s^{2}=g_{i j}(r, \theta) d x^{i} d x^{i}+h_{a b}(r, \theta, s) \delta y^{a} \delta y^{b},
$$

with diagonal coefficients

$$
g_{i j}=\left[\begin{array}{ccc}
1 & 0 & 0 \\
0 & g_{2} & 0 \\
0 & 0 & g_{3}
\end{array}\right] \quad \text { and } h_{a b}=\left[\begin{array}{cc}
h_{4} & 0 \\
0 & h_{5}
\end{array}\right]
$$

if instead the coordinate bases (8) and (9) one introduce the anholonomic frames (anisotropic bases)

$$
\delta_{\alpha} \equiv \frac{\delta}{d u^{\alpha}}=\left(\delta_{i}=\partial_{i}-N_{i}^{b}(u) \partial_{b}, \partial_{a}=\frac{\partial}{d y^{a}}\right)
$$

and

$$
\delta^{\alpha} \equiv \delta u^{\alpha}=\left(\delta^{i}=d x^{i}, \delta^{a}=d y^{a}+N_{k}^{a}(u) d x^{k}\right),
$$

where the $N$-coefficients are parametrized

$$
N_{1}^{4,5}=0, \quad N_{2,3}^{4}=w_{2,3} \quad \text { and } \quad N_{2,3}^{5}=n_{2,3}
$$

(they define an associated to some anholonomic frames (16) and (17), nonlinear connection, $N$-connection, structure, see details in Refs. [3,15-17,20]). A $N$-connection induces a global decomposition of the 5D pseudo-Riemannian spacetime into holonomic (horizontal, h) and anholonomic (vertical, v) directions. In a preliminary form the concept of $N$-connections was applied by E. Cartan in his approach to Finsler geometry [18] and a rigorous definition was given by Barthel [19] (Ref. [20] gives a modern approach to the geometry of $N$-connections, and to generalized Lagrange and Finsler geometry, see also Ref. [16] for applications of $N$-connection formalism in supergravity and superstring theory). As a particular case one obtains the linear connections if $N_{i}^{a}(x, y)=\Gamma_{b i}^{a}(x) y^{a}$.

A quite surprising result is that the $N$-connection structures can be naturally defined on (pseudo)-Riemannian spacetimes $[3,15,16]$ by associating them with some anholonomic frame fields (vielbeins) of type (16) satisfying the relations $\delta_{\alpha} \delta_{\beta}-\delta_{\beta} \delta_{\alpha}=W_{\alpha \beta}^{\gamma} \delta_{\gamma}$, with nontrivial anholonomy coefficients

$$
\begin{aligned}
& W_{i j}^{k}=0, \quad W_{a j}^{k}=0, \quad W_{i a}^{k}=0, \quad W_{a b}^{k}=0, \quad W_{a b}^{c}=0, \\
& W_{i j}^{a}=-\Omega_{i j}^{a}, \quad W_{b j}^{a}=-\partial_{b} N_{j}^{a}, \quad W_{i a}^{b}=\partial_{a} N_{j}^{b},
\end{aligned}
$$


where

$$
\Omega_{i j}^{a}=\delta_{j} N_{i}^{a}-\delta_{i} N_{j}^{a}
$$

is the nonlinear connection curvature ( $N$-curvature).

One says that the $N$-connection coefficients model a locally anisotropic structure on spacetime (a locally anisotropic spacetime) when the partial derivative operators and coordinate differentials, (8) and (9), are, respectively, changed into $N$-elongated operators (16) and (17).

Conventionally, the $N$-coefficients decompose the spacetime values (tensors, spinors and connections) into sets of mixed holonomic-anholonomic variables (coordinates) provided, respectively, with 'holonomic' indices of type $i, j, k, \ldots$ and with 'anholonomic' indices of type $a, b, c, \ldots$ Tensors, metrics and linear connections with coefficients defined with respect to anholonomic frames (16) and (17) are distinguished (d) by $N$-coefficients into holonomic and anholonomic subsets and called, in brief, d-tensors, d-metrics and d-connections.

\subsection{D-connections, $d$-torsions and d-curvatures}

On (pseudo)-Riemannian spacetimes the associated $N$-connection structure can be treated as a "pure" anholonomic frame effect which is induced if we are dealing with mixed sets of holonomic-anholonomic basis vectors. When we are transferring our considerations only to coordinate frames (8) and (9) the $N$-connection coefficients are removed into both off-diagonal and diagonal components of the metric like in (11). In some cases the $\mathrm{N}$-connection (anholonomic) structure is to be stated in a non-dynamical form by definition of some initial (boundary) conditions for the frame structure, following some prescribed symmetries of the gravitational-matter field interactions, or, in another cases, a subset of $N$-coefficients have to be treated as some dynamical variables defined as to satisfy the Einstein equations.

\subsubsection{D-metrics and d-connections}

A metric of type (14), in general, with arbitrary coefficients $g_{i j}\left(x^{k}, y^{a}\right)$ and $h_{a b}\left(x^{k}, y^{a}\right)$ defined with respect to a $N$-elongated basis (17) is called a d-metric.

A linear connection $D_{\delta_{\gamma}} \delta_{\beta}=\Gamma_{\beta \gamma}^{\alpha}(x, y) \delta_{\alpha}$, associated to an operator of covariant derivation $D$, is compatible with a metric $g_{\alpha \beta}$ and $N$-connection structure on a $5 \mathrm{D}$ pseudo-Riemannian spacetime if $D_{\alpha} g_{\beta \gamma}=0$. The linear d-connection is parametrized by irreducible h-v-components, $\Gamma^{\alpha}{ }_{\beta \gamma}=\left(L^{i}{ }_{j k}, L^{a}{ }_{b k}, C^{i}{ }_{j c}, C^{a}{ }_{b c}\right)$, where

$$
\begin{aligned}
& L_{j k}^{i}=\frac{1}{2} g^{i n}\left(\delta_{k} g_{n j}+\delta_{j} g_{n k}-\delta_{n} g_{j k}\right), \\
& L_{b k}^{a}=\partial_{b} N_{k}^{a}+\frac{1}{2} h^{a c}\left(\delta_{k} h_{b c}-h_{d c} \partial_{b} N_{k}^{d}-h_{d b} \partial_{c} N_{k}^{d}\right), \\
& C^{i}{ }_{j c}=\frac{1}{2} g^{i k} \partial_{c} g_{j k}, \quad C_{b c}^{a}=\frac{1}{2} h^{a d}\left(\partial_{c} h_{d b}+\partial_{b} h_{d c}-\partial_{d} h_{b c}\right) .
\end{aligned}
$$

This defines a canonical linear connection (distinguished by a $N$-connection) which is similar to the metric connection introduced by Christoffel symbols in the case of holonomic bases. 


\subsubsection{D-torsions and d-curvatures}

The anholonomic coefficients $w_{\alpha \beta}^{\gamma}$ and $N$-elongated derivatives give nontrivial coefficients for the torsion tensor, $T\left(\delta_{\gamma}, \delta_{\beta}\right)=T^{\alpha}{ }_{\beta \gamma} \delta_{\alpha}$, where

$$
T_{\beta \gamma}^{\alpha}=\Gamma_{\beta \gamma}^{\alpha}-\Gamma_{\gamma \beta}^{\alpha}+w_{\beta \gamma}^{\alpha},
$$

and for the curvature tensor, $R\left(\delta_{\tau}, \delta_{\gamma}\right) \delta_{\beta}=R_{\beta}{ }^{\alpha}{ }_{\gamma \tau} \delta_{\alpha}$, where

$$
R_{\beta}^{\alpha}{ }_{\gamma \tau}=\delta_{\tau} \Gamma_{\beta \gamma}^{\alpha}-\delta_{\gamma} \Gamma_{\beta \tau}^{\alpha}+\Gamma_{\beta \gamma}^{\varphi} \Gamma_{\varphi \tau}^{\alpha}-\Gamma \varphi_{\beta \tau} \Gamma_{\varphi \gamma}^{\alpha}+\Gamma_{\beta \varphi}^{\alpha} w_{\gamma \tau}^{\varphi} .
$$

We emphasize that the torsion tensor on (pseudo)-Riemannian spacetimes is induced by anholonomic frames, whereas its components vanish with respect to holonomic frames. All tensors are distinguished (d) by the $N$-connection structure into irreducible h-vcomponents, and are called d-tensors. For instance, the torsion, d-tensor has the following irreducible, non-vanishing, h-v-components, $T^{\alpha}{ }_{\beta \gamma}=\left\{T_{j k}^{i}, C^{i}{ }_{j a}, S^{a}{ }_{b c}, T^{a}{ }_{i j}, T^{a}{ }_{b i}\right\}$, where

$$
\begin{aligned}
& T_{. j k}^{i}=T_{j k}^{i}=L_{j k}^{i}-L_{k j}^{i}, \quad T_{j a}^{i}=C_{. j a}^{i}, \quad T_{a j}^{i}=-C_{j a}^{i}, \\
& T_{. j a}^{i}=0, \quad T_{. b c}^{a}=S_{. b c}^{a}=C_{b c}^{a}-C_{c b}^{a}, \\
& T_{. j j}^{a}=-\Omega_{i j}^{a}, \quad T_{. b i}^{a}=\partial_{b} N_{i}^{a}-L_{. b i}^{a}, \quad T_{. i b}^{a}=-T_{. b i}^{a}
\end{aligned}
$$

(the d-torsion is computed by substituting the h-v-components of the canonical dconnection (19) and anholonomic coefficients (18) into the formula for the torsion coefficients (20)). The curvature d-tensor has the following irreducible, non-vanishing, hv-components $R_{\beta}{ }^{\alpha}{ }_{\gamma \tau}=\left\{R_{h . j k}^{i}, R_{b . j k}^{a}, P_{j . k a}^{i}, P_{b . k a}^{. c}, S_{j . b c}^{i}, S_{b . c d}^{a}\right\}$, where

$$
\begin{aligned}
& R_{h . j k}^{. i}=\delta_{k} L_{. h j}^{i}-\delta_{j} L_{. h k}^{i}+L_{. h j}^{m} L_{m k}^{i}-L_{. h k}^{m} L_{m j}^{i}-C_{. h a}^{i} \Omega_{. j k}^{a}, \\
& R_{b . j k}^{. a}=\delta_{k} L_{. b j}^{a}-\delta_{j} L_{. b k}^{a}+L_{. b j}^{c} L_{. c k}^{a}-L_{. b k}^{c} L_{. c j}^{a}-C_{. b c}^{a} \Omega_{. j k}^{c}, \\
& P_{j . k a}^{. i}=\partial_{a} L_{. j k}^{i}+C_{. j b}^{i} T_{. k a}^{b}-\left(\delta_{k} C_{. j a}^{i}+L_{. l k}^{i} C_{. j a}^{l}-L_{. j k}^{l} C_{. l a}^{i}-L_{. a k}^{c} C_{. j c}^{i}\right), \\
& P_{b . k a}^{. c}=\partial_{a} L_{. b k}^{c}+C_{. b d}^{c} T_{. k a}^{d}-\left(\delta_{k} C_{. b a}^{c}+L_{. d k}^{c} C_{. b a}^{d}-L_{. b k}^{d} C_{. d a}^{c}-L_{. a k}^{d} C_{. b d}^{c}\right), \\
& S_{j . b c}^{i}=\partial_{c} C_{. j b}^{i}-\partial_{b} C_{. j c}^{i}+C_{. j b}^{h} C_{. h c}^{i}-C_{. j c}^{h} C_{h b}^{i}, \\
& S_{b . c d}^{a}=\partial_{d} C_{. b c}^{a}-\partial_{c} C_{. b d}^{a}+C_{. b c}^{e} C_{. e d}^{a}-C_{. b d}^{e} C_{. e c}^{a}
\end{aligned}
$$

(the d-curvature components are computed in a similar fashion by using the formula for curvature coefficients (21)).

\section{Einstein equations with anholonomic variables}

In this section we write and analyze the Einstein equations on 5D (pseudo)-Riemannian spacetimes provided with anholonomic frame structures and associated $N$-connections. 


\subsection{Einstein equations with matter sources}

The Ricci tensor $R_{\beta \gamma}=R_{\beta}{ }^{\alpha}{ }_{\gamma \alpha}$ has the d-components

$$
\begin{aligned}
& R_{i j}=R_{i . j k}^{. k}, \quad R_{i a}=-{ }^{2} P_{i a}=-P_{i . k a}^{. k}, \\
& R_{a i}={ }^{1} P_{a i}=P_{a . i b}^{. b}, \quad R_{a b}=S_{a . b c}^{. c} .
\end{aligned}
$$

In general, since ${ }^{1} P_{a i} \neq{ }^{2} P_{i a}$, the Ricci d-tensor is non-symmetric (this could be with respect to anholonomic frames of reference). The scalar curvature of the metric dconnection, $\overleftarrow{R}=g^{\beta \gamma} R_{\beta \gamma}$, is computed as

$$
\overleftarrow{R}=G^{\alpha \beta} R_{\alpha \beta}=\widehat{R}+S
$$

where $\widehat{R}=g^{i j} R_{i j}$ and $S=h^{a b} S_{a b}$.

By substituting (23) and (24) into the 5D Einstein equations

$$
R_{\alpha \beta}-\frac{1}{2} g_{\alpha \beta} R=\kappa \Upsilon_{\alpha \beta},
$$

where $\kappa$ and $\Upsilon_{\alpha \beta}$ are, respectively, the coupling constant and the energy-momentum tensor. The definition of matter sources with respect to anholonomic frames is considered in Ref. [3].

\section{2. $5 D$ vacuum Einstein equations}

In this paper we deal only with vacuum 5D, locally, anisotropic gravitational equations which in invariant $\mathrm{h}-\mathrm{v}$-components are written

$$
\begin{aligned}
& R_{i j}-\frac{1}{2}(\widehat{R}+S) g_{i j}=0, \\
& S_{a b}-\frac{1}{2}(\widehat{R}+S) h_{a b}=0, \\
& { }^{1} P_{a i}=0, \quad{ }^{2} P_{i a}=0 .
\end{aligned}
$$

The main 'trick' of the anholonomic frames method for integrating the Einstein equations in general relativity and various (super)-string and higher/lower-dimensional gravitational theories is to find the coefficients $N_{j}^{a}$ such that the block matrices $g_{i j}$ and $h_{a b}$ are diagonalized $[3,16]$. This greatly simplifies computations. With respect to such anholonomic frames the partial derivatives are $N$-elongated (locally anisotropic).

\subsubsection{Non-trivial Ricci components}

The metric (10) with coefficients (11) (equivalently, the d-metric (14) with coefficients (15)) is assumed to solve the 5D Einstein vacuum equations $R_{\alpha \beta}=0$, which are distinguished in $\mathrm{h}$ - and v-components as

$$
\begin{aligned}
& R_{2}^{2}=R_{3}^{3}=\frac{-1}{2 g_{2} g_{3}}\left[g_{3}^{\bullet \bullet}-\frac{g_{2}^{\bullet} g_{3}^{\bullet}}{2 g_{2}}-\frac{\left(g_{3}^{\bullet}\right)^{2}}{2 g_{3}}+g_{2}^{\prime \prime}-\frac{g_{2}^{\prime} g_{3}^{\prime}}{2 g_{3}}-\frac{\left(g_{2}^{\prime}\right)^{2}}{2 g_{2}}\right]=0, \\
& R_{4}^{4}=R_{5}^{5}=-\frac{\beta}{2 h_{4} h_{5}}=0,
\end{aligned}
$$




$$
\begin{aligned}
& R_{4 i}=-w_{i} \frac{\beta}{2 h_{5}}-\frac{\alpha_{i}}{2 h_{5}}=0, \quad i=2,3, \\
& R_{5 i}=-\frac{h_{5}}{2 h_{4}}\left[n_{i}^{* *}+\gamma n_{i}^{*}\right]=0, \quad i=2,3,
\end{aligned}
$$

where

$$
\begin{aligned}
& \alpha_{2}=h_{5}^{* \bullet}-\frac{h_{5}^{*}}{2}\left(\frac{h_{4}^{\bullet}}{h_{4}}+\frac{h_{5}^{\bullet}}{h_{5}}\right)=h_{5}^{*}\left(\ln \left|f_{5}^{*}\right|+f_{5}-f_{4}\right)^{\bullet}, \quad h_{5}^{*} \neq 0, \\
& \alpha_{3}=h_{5}^{* \prime}-\frac{h_{5}^{*}}{2}\left(\frac{h_{4}^{\prime}}{h_{4}}+\frac{h_{5}^{\prime}}{h_{5}}\right)=h_{5}^{*}\left(\ln \left|f_{5}^{*}\right|+f_{5}-f_{4}\right)^{\prime}, \quad h_{5}^{*} \neq 0, \\
& \beta=h_{5}^{* *}-\frac{\left(h_{5}^{*}\right)^{2}}{2 h_{5}}-\frac{h_{5}^{*} h_{4}^{*}}{2 h_{4}}=h_{5}^{*}\left(\ln \left|f_{5}^{*}\right|+f_{5}-f_{4}\right)^{*}, \quad h_{5}^{*} \neq 0, \\
& \gamma=\frac{3}{2} \frac{h_{5}^{*}}{h_{5}}-\frac{h_{4}^{*}}{h_{4}}=\left[3 f_{5}-2 f_{4}\right]^{*},
\end{aligned}
$$

for further applications we gave the formulas with respect to $h_{4,5}$ coefficients of metric as well with respect to $f_{4,5}$, see (13), and, for simplicity, the partial derivatives are denoted $h^{\bullet}=\partial h / \partial x^{2}, f^{\prime}=\partial f / \partial x^{3}$ and $f^{*}=\partial f / \partial s$.

It was possible to construct very general classes of solutions for such equations [3-5] describing locally anisotropic soliton, black hole, black tori and wormhole objects.

\subsubsection{General properties of anisotropic vacuum solutions}

In the vacuum case Eqs. (27), (28), (29) and (30) form a very simplified system of equations with separations of variables which can be solved consequently for every couples of d-metric coefficients, $\left(g_{2}, g_{3}\right),\left(h_{4}, h_{5}\right)$, and $N$-connection coefficients $w_{2,3}$ and $n_{2,3}$ (see Ref. [10] on the main theorems and methods of constructing exact solutions):

1. Eq. (27) relates two functions $g_{2}(r, \theta)$ and $g_{3}(r, \theta)$ and their partial derivatives on 'isotropic' coordinates $r$ and $\theta$. The solution is trivial if we chose $g_{2}=1$ and $g_{3}=r^{2}$ in order to reduce the coefficients from (11), respectively, to those from (7).

2. Eq. (28) contains partial derivatives only on anisotropic coordinate $s$ and relates two functions $h_{4}(r, \theta, s)$ and $h_{5}(r, \theta, s)$. This equation is satisfied by arbitrary two functions $h_{4}(r, \theta, s)$ and $h_{5}(r, \theta)$ for which $h_{5}^{*}=0$.

If the condition $h_{5}^{*} \neq 0$ is satisfied, we can write (28), in $f$-variables (see (12)), as

$$
\left(\ln \left|f_{5}^{*}\right|+f_{5}-f_{4}\right)^{*}=0
$$

which is solved by arbitrary functions $f_{5}(r, \theta, s)$ and

$$
f_{4}=f_{4[0]}+\ln \left|f_{5}^{*}\right|+f_{5},
$$

where $f_{4[0]}=f_{4[0]}(r, \theta)$. The general solution of (28) expressing $h_{5}$ via $h_{4}$ is

$$
\begin{aligned}
h_{5} & =\left[h_{5[1]}(r, \theta)+h_{5[2]}(r, \theta) \int \sqrt{h_{4}(r, \theta, s)} d s\right]^{2} \\
& =h_{5[0]}(r, \theta)[1+\varpi(r, \theta) s]^{2}, \quad h_{4}^{*}=0,
\end{aligned}
$$


for some functions $f_{5[0,1,2]}(r, \theta)$ and $\varpi(r, \theta)$ stated by boundary conditions and locally anisotropic limits as well from the conditions that Eqs. (29) and (30) are compatible. Inversely, for a prescribed value of $h_{5}$, the general solution of (28) is (35) which can be rewritten with respect to variables $h_{4,5}$,

$$
\begin{aligned}
h_{4} & =h_{4[0]}(r, \theta)\left[\left(\sqrt{\left|h_{5}(r, \theta, s)\right|}\right)^{*}\right]^{2} \\
& =h_{4}(r, \theta, s) \text { an arbitrary function if } h_{5}^{*}=0,
\end{aligned}
$$

3. If the functions $h_{4}(r, \theta, s)$ and $h_{5}(r, \theta, s)$ were defined, Eq. (29) can be solved as independent linear algebraic equations for $w_{2,3}, w_{\hat{i}} \beta+\alpha_{\hat{i}}=0, \hat{i}=2,3$. For zero matter sources this is a trivial result because in this case the conditions $\beta=0$ and $\alpha_{\hat{i}}=$ 0 (see the formulas (31), (32) and (33)) are automatically fulfilled. In consequence, the resulting sourceless equations (29) became some trivial equations admitting arbitrary values of functions $w_{\hat{i}}(r, \theta, s)$; such functions can be associated to some coordinate transforms for vanishing anholonomy coefficients $W_{\alpha \beta}^{4}=0$, see (18), or to some anholonomy coefficients, in such cases being not contained in the vacuum Einstein equations, which must be stated by some boundary and symmetry conditions.

4. Eq. (29) can be solved in general form if the functions $h_{4}(r, \theta, s)$ and $h_{5}(r, \theta, s)$ (and, in consequence, the coefficient $\gamma$ from (34)) are known,

$$
\begin{aligned}
& n_{2,3}(r, \theta, s)=n_{2,3[0]}(r, \theta)+n_{2,3[1]}(r, \theta) \int \frac{h_{4}(r, \theta, s)}{h_{5}^{3 / 2}(r, \theta, s)} d s, \quad \gamma \neq 0, \\
& n_{2,3}(r, \theta, s)=n_{2,3[0]}(r, \theta)+n_{2,3[1]}(r, \theta) s, \quad \gamma=0,
\end{aligned}
$$

where the functions $n_{2,3[0]}(r, \theta)$ and $n_{2,3[1]}(r, \theta)$ should be defined from some boundary conditions.

\section{Taub-NUT metrics with anisotropies and running of constant}

The conformally transformed Taub-NUT metric (7) can be considered as a locally isotropic background with trivial vanishing local anisotropies. By coordinate transforms of the 5th coordinate and a conformal transform on two holonomic coordinates (here we mention that in two dimensions the coordinate and conformal transforms are equivalent) the isotropic background metric can be transformed into a form parameterizing the usual Taub-NUT solution of the vacuum Einstein equations.

The aim of this section is to construct and analyze three types of anisotropic generalizations of the Taub-NUT solution.

The data parameterizing a metric (11) (equivalently, a d-metric (14), or (7)) are

$$
\begin{aligned}
& x^{1}=t, \quad x^{2}=r, \quad x^{3}=\theta, \quad y^{4}=s=\varsigma, \quad y^{5}=p=\varphi, \\
& g_{1}=-1, \quad g_{2}=1, \quad g_{3}=r^{2}, \quad h_{4}=r^{2} \sin ^{2} \theta, \quad h_{5}=16 m_{0}^{2} V^{2}(r), \\
& w_{i}=0, \quad n_{1}=0, \quad n_{2}=0, \quad n_{3}=\cos \theta,
\end{aligned}
$$

which defines a trivial (locally isotropic) solution of the vacuum Einstein equations (27)(30) satisfying the conditions $h_{4,5}^{*}=0$. 


\subsection{Generalizations of Taub-NUT solutions to anisotropies and running of constant}

The simplest way to obtain anisotropic Taub-NUT-like solutions, is to follow the approach developed for generation anisotropic black hole [3,15] and wormhole/flux tube solutions [4] when the constants like mass and charges are considered to be effectively anisotropically polarized by some anholonomic (anisotropic) higher-dimensional interactions; in our case is to consider that the parameter $m_{0}$ from (7) (see also (38)) is not a constant but a renormalized value $m_{0} \rightarrow m=m(r, \theta, s)$.

\subsubsection{Taub-NUT metrics with anisotropic running of constants on $s=5$}

We generate from the isotropic solution (38) a new one, anisotropic, in this manner. Let us consider the case when $h_{4}(r, \theta)=r^{2} \sin ^{2} \theta$, when $h_{4}^{*}=0$, but $h_{5}^{*} \neq 0$. Following the solution (36) we parameterize

$$
16 m^{2}(r, \theta, \varsigma)=16 m_{0}^{2}[1+\varpi(r, \theta) \varsigma]^{2}
$$

and

$$
h_{5}(r, \theta, \varsigma)=16 m_{0}^{2} V^{2}(r)[1+\varpi(r, \theta) \varsigma]^{2},
$$

where $\widehat{V}(r, \theta, \varsigma)$ is just the function (2) but defined by a renormalized value $m(r, \theta, \varsigma)$.

The conditions of vanishing of constants (31) and (32) are also satisfied if $\varpi(\varsigma, \theta)=$ $\varpi_{0}=$ const. For simplicity, in this subsection, we shall consider solutions with "pure" running of the constant $m$ on $\zeta$ and the function

$$
\widehat{V}(r, \varsigma)=\left(1+\frac{4 m(\varsigma)}{r}\right)^{-1} .
$$

Because (in this case) the coefficients $\beta$ and $\alpha_{2,3}$, and in consequence. $w_{2,3}$ could be arbitrary functions (see formulas (31), (32) and (33) and Eq. (29)); in the locally isotropic limit, $\varpi \chi \rightarrow 0$, we put that $w_{2,3} \rightarrow 0$. The values $n_{2,3}$ depends on anisotropic variable $\varsigma$ as follow from the solution (38) with $\gamma=3 \varpi_{0}$.

We obtain the locally isotropic limit (38), for $\varpi \varsigma \rightarrow 0$, if we fix the boundary conditions with $n_{2[0,1]}=0, n_{3[0]}=0$ but $n_{3[1]}(r, \theta)=\cos \theta$.

So, a parametrization of the ansatz (11),

$$
\begin{aligned}
\delta s^{2}= & -d t^{2}+d r^{2}+r^{2} d \theta^{2}+r^{2} \sin ^{2} \theta d \varsigma^{2} \\
& +16 m_{0}^{2}[1+\varpi(r, \theta) \varsigma]^{2} \widehat{V}^{2}(r, \varsigma)\left[d \varphi+\cos \theta \exp \left(3 \varpi_{0} \varsigma\right) d \theta\right]^{2},
\end{aligned}
$$

defines a locally anisotropic solution of the vacuum Einstein equations (27)-(30) generalizing the Taub-NUT solution (7). We can treat the solution (41) as describing an anisotropic Kaluza-Klein monopole with running constant (on extra-dimensional coordinate) obtained by embedding the anisotropic Taub-NUT gravitational instanton into 5D theory, adding the coordinate in a way as to be compatible with running of constant of effective magnetic configurations (in brief, we shall call such solutions as $\varsigma$-solutions).

We conclude that the solutions describing gravitational monopoles and instantons [12, 14] can be generalized to some anisotropic configurations with running constants. 


\subsubsection{Taub-NUT metric with anisotropy of constants on angle $\varphi$}

In a similar fashion we can consider anisotropic (angular) dependencies of constants with $s=\varphi$ (in brief, we call such solutions as $\varphi$-solutions). The simplest way is to take $h_{5}^{*}=0$ but $h_{4}^{*} \neq 0$, i.e., to define a solution with

$$
\begin{aligned}
& h_{4}(r, \theta, \varphi)=16 m_{0}^{2} \exp [\varpi(r, \theta, \varphi)] \widehat{V}^{2}(r, \theta, \varphi), \\
& h_{5}(r, \theta)=r^{2} \sin ^{2} \theta,
\end{aligned}
$$

where $\widehat{V}(r, \theta, \varphi)$ is the function (2) but defined by the renormalized parameter $m(r, \theta, \varphi)$

$$
\widehat{V}(r, \theta, \varphi)=\left(1+\frac{4 m(r, \theta, \varphi)}{r}\right)^{-1} ;
$$

we can take arbitrary values of $w_{2,3}$ because $\beta=0$ and $\alpha_{\hat{i}}$ from Eq. (29) vanish.

For small, constant, polarizations we can approximate

$$
h_{4}(r, \theta, \varphi)=16 m_{0}^{2} V^{2}(r)[1+\varpi \varphi]
$$

and consider only anisotropic angular variations of the constant, $m \sim m(\varphi)$.

The d-metric

$$
\begin{gathered}
\delta s^{2}=-d t^{2}+d r^{2}+r^{2} d \theta^{2}+16 m_{0}^{2} \exp [\varpi(r, \theta, \varphi)] \widehat{V}^{2}(r, \theta, \varphi)(d \varphi+\cos \theta d \theta)^{2} \\
+r^{2} \sin ^{2} \theta\left[d \varsigma+n_{2[1]}(r, \theta) d r \int \varpi(r, \theta, \varphi) d \varphi\right. \\
\left.+n_{3[1]}(r, \theta) d \theta \int \varpi(r, \theta, \varphi)\right]^{2},
\end{gathered}
$$

models a locally anisotropic generalization of the solution (7) for anisotropic dependencies of the constant $m$ on angle $\varphi$ which describe a 5D Kaluza-Klein monopole with angular anisotropic constant obtained by embedding the anisotropic Taub-NUT gravitational instanton into 5D theory.

So, in this subsection, we constructed two classes of generalized, anisotropic, TaubNUT solutions of the 5D vacuum Einstein equations: the first class is for $s=\varphi$ (i.e., anisotropic polarizations) and the second is for $s=\varsigma$ (i.e., with dependence of the constant $m$ on the fifth coordinate). The metric (11), describing these two classes of solutions, can be written with respect to a coordinate frame (8) where the existence of non-diagonal terms is emphasized.

\subsection{Gravitational $\theta$-elliptic polarizations of the Taub-NUT solutions}

This subsection is devoted to such generalizations of the Taub-NUT metrics when the anisotropies of constant are modeled by elliptic polarizations on the angle $\theta$, i.e., the constant is renormalized as $m_{0} \rightarrow m(\theta, \varsigma)($ or $m(\theta, \varphi)) \sim m_{1}\left(1+\varepsilon_{r} \cos \theta\right)^{-1}$, where $\varepsilon_{r}$ is the eccentricity of an ellipse and $m_{1}=m_{1}(\varsigma)$ (or $m_{1}=m_{1}(\varphi)$ ) is defined as for the solution (41), in brief, for $\varsigma$-solutions (or as for the solution (43), in brief, for $\varphi$-solution). Because the method of construction of such solutions is very similar to that considered in the previous subsection, we shall omit the details on integration of equations and present 
only the final values of the d-metric and $N$-connection coefficients. The results can be verified by straightforward calculations.

\subsubsection{Elliptic polarizations for 5 -solutions}

The simplest way of definition of such polarizations for $\varsigma$-solutions (36) and (39) is to consider the off-diagonal metric

$$
\begin{aligned}
\delta s^{2}=- & d t^{2}+d r^{2}+r^{2} d \theta^{2}+r^{2} \sin ^{2} \theta d \varsigma^{2} \\
+ & 16 m_{0}^{2}\left[1+\varepsilon_{r} \cos \theta\right]^{-2} \\
& \times[1+\varpi(r, \theta) \varsigma]^{2} \widehat{V}^{2}(r, \theta, \varsigma)\left[d \varphi+\cos \theta \exp \left(3 \varpi_{0} \varsigma\right) d \theta\right]^{2},
\end{aligned}
$$

the function $\widehat{V}(r, \theta, \varsigma)$ is the function (2) redefined by an ellipsoidally renormalized coefficient $m \sim m_{0}\left[1+\varepsilon_{r} \cos \theta\right]^{-1}$,

$$
\widehat{V}(r, \theta, \varsigma)=\left(1+\frac{4 m_{0}[1+\varpi(r, \theta) \varsigma]}{r\left(1+\varepsilon_{r} \cos \theta\right)}\right)^{-1},
$$

where there is also a linear dependence on extra-dimensional coordinate $\varsigma$; for simplicity, we can choose $\varpi(r, \theta)=\varpi_{0}=$ const.

\subsubsection{Elliptic polarizations for $\varphi$-solutions}

Such $\varphi$-solutions with elliptic variations of the constant $m$ on angle $\theta$ are distinguished by the metric

$$
\begin{aligned}
& \delta s^{2}=- d t^{2}+d r^{2}+r^{2} d \theta^{2} \\
&+ 16 m_{0}^{2}\left[1+\varepsilon_{r} \cos \theta\right]^{-2} \exp [\varpi(r, \theta, \varphi)] \widehat{V}^{2}(r, \theta, \varphi)(d \varphi+\cos \theta d \theta)^{2} \\
&+r^{2} \sin ^{2} \theta\left[d \varsigma+n_{2[1]}(r, \theta) d r \int \varpi(r, \theta, \varphi) d \varphi\right. \\
&\left.+n_{3[1]}(r, \theta) d \theta \int \varpi(r, \theta, \varphi)\right]^{2},
\end{aligned}
$$

where $\widehat{V}(r, \theta, \varphi)$ generalize the functions (40), (42) for anisotropic dependencies like

$$
\begin{aligned}
& m(r, \theta, \varphi) \sim m_{1}(\varphi)\left[1+\varepsilon_{r} \cos \theta\right]^{-1}, \\
& \widehat{V}(r, \theta, \varphi)=\left(1+\frac{4 m_{1}(\varphi)}{r\left[1+\varepsilon_{r} \cos \theta\right]}\right)^{-1} .
\end{aligned}
$$

The constructed solutions of 5D vacuum Einstein equations, (44) and (45) contain some additional elliptic polarizations comparing with respective solutions (41) and (43). Such 5D Kaluza-Klein monopoles induced from 4D Taub-NUT instantons behave theirselves as some objects with running on $\zeta$, or anisotropic on $\varphi$, constant $m$ which is also elliptically polarized on the angle $\theta$. 


\section{A superposition of wormhole and Taub-NUT metrics}

By applying the method of anholonomic frames we can construct nonlinear anisotropic superposition of the Taub-NUT metric with some metrics defining wormhole/flux tube configurations: this way one defines anisotropic generalizations of the metric (1) which are called anisotropic Taub-NUT wormholes. For simplicity, in this paper we consider only a $\varsigma$-solution for the Taub-NUT configurations with the wormhole background chosen as to be locally isotropic (see Ref. [13], on isotropic wormholes and flux tubes, and [4], on locally anisotropic wormhole solutions).

For our purpose the ansatz (11) is generalized by introducing the coefficient $N_{1}^{6}=$ $n_{1}(r)=\omega(r)$ of the nonlinear connection and, for simplicity, we consider $w_{i}=0$ and $n_{2}=0$

$$
\left[\begin{array}{ccccc}
-1+n_{1}{ }^{2} h_{5} & 0 & n_{1} n_{3} h_{5} & 0 & n_{1} h_{5} \\
0 & g_{2} & 0 & 0 & 0 \\
n_{1} n_{3} h_{5} & 0 & g_{3}+n_{3}{ }^{2} h_{5} & 0 & n_{3} h_{5} \\
0 & 0 & 0 & h_{4} & 0 \\
n_{1} h_{5} & 0 & n_{3} h_{5} & 0 & h_{5}
\end{array}\right]
$$

which results in a diagonal d-metric

$$
\begin{aligned}
& \delta s^{2}=-d t^{2}+g_{2}(r, \theta) d r^{2}+g_{3}(r, \theta) d \theta^{2}+h_{4}(r, \theta, \varsigma) \delta \varsigma^{2}+h_{5}(r, \theta, \varsigma) \delta \varphi^{2}, \\
& \delta \varsigma=d \varsigma, \quad \delta \varphi=d \varphi+n_{1}(r) d t+\left(1+n_{0}\right) \cos \theta d \theta,
\end{aligned}
$$

where the coefficients $n_{1}(r)=\omega(r)$ and $n_{3}(\theta)=\left(1+n_{0}\right) \cos \theta$ and the constant $n_{0}$ are introduced for the $t$ and $\theta$ components of the wormhole electromagnetic potential like in (1).

The data defining a vacuum $\varsigma$-solution for the (46), including the solution (41) into a wormhole background are given by the coefficients of the d-metric

$$
\begin{aligned}
\delta s^{2}=- & d t^{2}+d r^{2}+a(r) d \theta^{2}+a(r) \sin ^{2} \theta d \varsigma^{2} \\
+ & 16 m_{0}^{2} r_{0}^{2} \exp [2 \psi(r)][1+\varpi(r, \theta) \varsigma]^{2} \widehat{V}^{2}(r, \varsigma) \\
& \times\left[d \varphi+\omega(r) d r+\left(1+n_{0}\right) \cos \theta \exp \left(3 \varpi_{0} \varsigma\right) d \theta\right]^{2},
\end{aligned}
$$

where $\widehat{V}(r, \varsigma)$ and $V(r)$ are, respectively, those from (40) and (2) and the constant $r_{0}^{2}$ and term $\exp [2 \psi(r)]$ were introduced as some multiples defining the wormhole/flux tube configuration.

The properties of the Taub-NUT monopole with running constant $m(\varsigma)$ are the same as those stated for the solution (41), resulting in a similar to (3) magnetic field components

$$
\begin{aligned}
& A_{r}^{(\text {nut })}=A_{\varphi}^{(\text {nut })}=0, \quad A_{\theta}^{(\text {nut })}=4 m(\varsigma)(1-\cos \theta), \\
& \vec{B}^{\text {(nut) }}=\operatorname{rot} \vec{A}^{(\text {nut })}=\frac{4 m(\varsigma) \vec{r}}{r^{3}} .
\end{aligned}
$$

embedded into a static background of wormhole/flux tube configurations defined by additional components of electric, $E_{\mathrm{KK}}^{(\text {worm })}=q_{0} / a(r)$, and magnetic, $H_{\mathrm{KK}}^{(\text {worm })}=Q_{0} / a(r)$, fields as it is defined, respectively, by the formulas (4) and (5). 
As the free parameters of the wormhole background (see Ref. [13]) are varied there are five classes of solutions with the properties:

1. $Q=0$ or $H_{\mathrm{KK}}^{\text {(worm) }}=0$, a wormhole-like 'electric' object;

2. $q=0$ or $E_{\mathrm{KK}}^{(\text {worm })}=0$, a finite 'magnetic' flux tube;

3. $H_{\mathrm{KK}}^{(\text {worm })}=E_{\mathrm{KK}}^{\text {(worm) }}$, an infinite 'electromagnetic' flux tube;

4. $H_{\mathrm{KK}}^{\text {(worm) }}<E_{\mathrm{KK}}^{\text {(worm) }}$, a wormhole-like 'electromagnetic' object;

5. $H_{\mathrm{KK}}^{(\text {worm })}>E_{\mathrm{KK}}^{(\text {worm })}$, a finite, 'magnetic-electric' flux tube.

The number wormhole/flux tube classes must be extended to new configurations which arise in the presence of the magnetic field of the Taub-NUT monopole, for instance, by considering the 6th class for the pure monopole configuration; we must take into account possible contribution of the monopole magnetic field to the structure of magnetic flux tubes, wormhole-like electromagnetic object and/or magnetic-electric flux tubes by analyzing the total magnetic field $H_{\mathrm{KK}}^{(\text {worm })}+B^{\text {(nut) }}$ with possible (elliptic and another type) vacuum gravitational polarizations.

Finally, in this section, we remark that in a similar fashion we can construct $\varphi$-solutions with an anisotropic parameter $m(\varphi)$ describing an anisotropic Taub-NUT monopole embedded into a wormhole/flux tube background and to generalized both $\varsigma$ - and $\varphi$-solutions for various configurations with elliptic polarizations and rotation hypersurface symmetries (like rotation ellipsoids, elliptic cylinders, bipolars and tori) as we constructed exact solutions of 3D-5D Einstein equations describing anisotropic black holes/tori and anisotropic wormholes [3-5].

\section{Anisotropically spinning particles and integrals of motion}

In the previous two sections we proved that the Taub-NUT and wormhole metrics admit various type of anisotropic generalizations modeled by anholonomic frames with associated $N$-connection structure. It is of interest the investigation of symmetries of such anisotropic spaces and definition of corresponding invariants of motion of spinning particles. The general rules for developing of corresponding geodesic calculus and definition of generalized anisotropic Killing vectors are:

- We have to change the partial derivatives and differentials into $N$-elongated ones (8) and (9) by redefinition of usual formulas for developing a formalism of differential, integral and variational calculus on anisotropic spaces.

- The metric, linear connection, curvature and Ricci tensors have to be changed into respective d-objects (d-metric (14), d-connection (19) and corresponding curvature and Ricci d-tensors); the d-torsion on (pseudo)-Riemannian spaces should be treated as an anholonomic frame effect which vanishes with respect to coordinate bases.

- The Greek indices $\alpha, \beta, \ldots$ should be split into horizontal $(i, j, \ldots)$ and vertical $(a, b, \ldots)$ ones which on necessity will point to some holonomic-anholonomic character of variables. 
- By using d-metrics and d-connections the differential, integral and variational calculus on Riemannian manifolds is adapted to the anholonomic frame structure with associated $N$-connection. With respect to $N$-adapted frames (8) and (9) the geometry is similar to the usual Riemannian one when the anholonomic (constrained dynamics) is coded into the coefficients of $N$-connection modeling a local anisotropy.

- As a matter of principle, all constructions defined with respect to anholonomic bases can be removed with respect to usual coordinate frames, but in this case the metrics became generically off-diagonal and a number of symmetries (and their constraints) of manifolds are hidden in rather sophisticate structures and relations for redefined holonomic objects.

\subsection{Moving of spinless particles in anisotropic Taub-NUT spaces}

\subsubsection{Killing $d$-vectors}

The geodesic motion of a spinless particle of unit mass moving into a background stated by a d-metric $g_{\alpha \beta}=\left(g_{i j}, h_{a b}\right)$ (see (14)) can be derived from the action:

$$
\begin{aligned}
S & =\frac{1}{2} \int_{a}^{b} d \tau g_{\mu \nu}(u) \frac{d u}{d \tau^{\mu}} \frac{d u}{d \tau^{\nu}} \\
& =\frac{1}{2} \int_{a}^{b} d \tau\left[g_{i j}(x, y) \frac{d x}{d \tau^{i}} \frac{d x}{d \tau^{j}}+h_{a b}(x, y) \frac{d y}{d \tau^{a}} \frac{d y}{d \tau^{a}}\right],
\end{aligned}
$$

where $\tau$ is a parameter.

The invariance of d-metrics defining anisotropic generalizations of Taub-NUT metrics under spatial rotations and $\varsigma$ translations is generated by four Killing d-vectors which are obtained by anholonomic transforms of the usual Killing vectors into corresponding one with elongated partial derivatives $\partial_{\mu} \rightarrow \delta_{\mu}=\left(\partial_{i}, \delta_{a}\right)$, where the partial derivative on the new 5 th coordinate, are $\frac{\partial}{\partial \varsigma}=\frac{\partial \chi}{\partial \varsigma} \frac{\partial}{\partial \chi}$, see (6). We write the Killing d-vectors as

$$
D^{(\alpha)}=R^{(\alpha) \mu} \delta_{\mu}=R^{(\alpha) i} \partial_{i}+R^{(\alpha) a} \delta_{a}, \quad \alpha=1, \ldots, 4,
$$

or, in details,

$$
\begin{aligned}
& D^{(1)}=-\sin \varphi \frac{\delta}{\partial \theta}-\cos \varphi \cot \theta \frac{\partial}{\partial \varphi}+\frac{\cos \varphi}{\sin \theta} \frac{\partial \varsigma}{\partial \chi} \frac{\partial}{\partial \varsigma}, \\
& D^{(2)}=\cos \varphi \frac{\delta}{\partial \theta}-\sin \varphi \cot \theta \frac{\partial}{\partial \varphi}+\frac{\sin \varphi}{\sin \theta} \frac{\partial \varsigma}{\partial \chi} \frac{\partial}{\partial \varsigma}, \\
& D^{(3)}=\frac{\partial}{\partial \varphi}, \quad D^{(4)}=\frac{\partial \varsigma}{\partial \chi} \frac{\partial}{\partial \varsigma},
\end{aligned}
$$

following general considerations we give the formulas for arbitrary transforms of the 5 th coordinate, $\varsigma \rightarrow \varsigma(\chi, \ldots)$, and elongations of derivatives of type $\delta / \partial \theta=\partial / \partial \theta-n_{3} \partial / \partial \varsigma$. 


\subsubsection{Energy and momentum d-vector}

We know that in the purely locally isotropic bosonic case such invariances correspond to conservation of angular momentum and "relative electric charge" [9,21]. For anisotropic Taub-NUTS we can define similar objects by using anholonomic transforms of the values given with respect to coordinate bases into the corresponding values with anisotropic coefficients and variables given with respect to anholonomic frames:

$$
\begin{aligned}
& \vec{j}=\vec{r} \times \vec{p}+q \frac{\vec{r}}{r}, \quad \text { for } \quad s=\varsigma(\text { or } s=\varphi), \\
& q=16 m^{2}(r, \theta, s) V^{2}(r, \theta, s)\left(\frac{\partial \chi}{\partial \varsigma} \frac{d \varsigma}{d \tau}+\cos \theta \frac{d \theta}{d \tau}\right),
\end{aligned}
$$

where $\vec{p}=d \vec{r} / d \tau$ is the "mechanical momentum" which is only part of the momentum canonically conjugate to $\vec{r}$ (in anisotropization of the momentum formula we do not write the multiple $V^{-1}$ because the d-metric used for anisotropic constructions has its locally isotropic limit being multiplied on the $V$ as a conformal factor, see (7)). The energy is defined by

$$
\begin{aligned}
E & =\frac{1}{2} g^{\mu v} \Pi_{\mu} \Pi_{v}=\frac{1}{2} g^{i j} \Pi_{i} \Pi_{j}+\frac{1}{2} h^{a b} \Pi_{a} \Pi_{b} \\
& =\frac{1}{2}\left[\vec{r}^{2}+\left(\frac{q^{2}(r, \theta, s)}{4 m^{2}(r, \theta, s)}\right)^{2}\right]
\end{aligned}
$$

is also conserved, $\Pi_{\mu}=\left(\Pi_{i}, \Pi_{a}\right)$ being the covariant momentum d-vector; this value variates with respect to coordinate frames but behaves itselve as a usual energy with respect to $N$-adapted frames.

\subsubsection{Runge-Lenz $d$-vector}

There is a conserved vector analogous to the Runge-Lenz vector of the Coulomb problem in the locally isotropic case $[9,21,22]$, which with respect to anholonomic frames with anisotropic variables in spite of the fact of complexity of anholonomic motion in the anisotropic Taub-NUT spaces defined in previous sections,

$$
\vec{K}=\vec{K}_{\mu \nu} \frac{d u^{\mu}}{d \tau} \frac{d u^{\nu}}{d \tau}=\vec{p} \times \vec{j}+\left(\frac{q^{2}}{4 m}-4 m E\right) \frac{\vec{r}}{r},
$$

which implies that the trajectories are anisotropic deformations of conic sections.

\subsection{Spinning of particles with respect to anholonomic frames}

The pseudo-classical limit of the Dirac theory of a spin- $1 / 2$ fermion in curved spacetime is described by the supersymmetric extension of the usual relativistic point-particle [23] (the theory of spinors on spaces with generic local anisotropy was developed in Ref. [17], see also some models of locally anisotropic supergravity and superstring theories in Ref. [16]).

In this work, the configuration space of spinning particles in anisotropic space (anisotropic spinning space) is an extension of an ordinary Riemannian manifold provided 
with an anholonomic frame and associated $N$-connection structure, parametrized by local coordinates $\left\{u^{\mu}=\left(x^{i}, y^{a}\right)\right\}$, to a graded manifold parametrized by local coordinates $\left\{u^{\mu}, \psi^{\mu}\right\}$, with the first set of variables being Grassmann-even (commuting) and the second set Grassmann-odd (anticommuting). We emphasize that in anholonomic spaces distinguished by a $N$-connection structure we must define spinor and Grassmann variables separately on h-subspace (with holonomic variables) and on v-subspace (with anholonomic variables).

\subsubsection{Action for anisotropically spinning particles}

The equation of motion of an anisotropically spinning particle on a autoparallel (geodesic) is derived from the action:

$$
\begin{array}{r}
S=\int d \tau\left(\frac{1}{2} g_{\mu \nu}(u) \frac{d u^{\mu}}{d \tau} \frac{d u^{\nu}}{d \tau}+\frac{i}{2} g_{\mu \nu}(u) \psi^{\mu} \frac{D \psi^{\nu}}{D \tau}\right) \\
=\frac{1}{2} \int d \tau\left(g_{i j}(u) \frac{d x^{i}}{d \tau} \frac{d x^{j}}{d \tau}+i g_{i j}(u) \psi^{i} \frac{D \psi^{j}}{D \tau}\right. \\
\left.+h_{a b}(u) \frac{d y^{a}}{d \tau} \frac{d y^{b}}{d \tau}+i h_{a b}(u) \psi^{a} \frac{D \psi^{b}}{D \tau}\right),
\end{array}
$$

where $i^{2}=-1$.

The corresponding world-line anholonomic Hamiltonian is given by:

$$
H=\frac{1}{2} g^{\mu v} \Pi_{\mu} \Pi_{v}=\frac{1}{2}\left(g^{i j} \Pi_{i} \Pi_{j}+h^{a b} \Pi_{a} \Pi_{b}\right),
$$

where $\Pi_{\mu}=g_{\mu \nu} \frac{d u^{\mu}}{d \tau}=\left\{g_{i j} \frac{d x^{j}}{d \tau}, h_{a b} \frac{d y^{b}}{d \tau}\right\}$ is the covariant momentum d-vector.

\subsubsection{Poisson-Dirac brackets}

For any integral (we use the term integral instead of the usual one, constant, because on anisotropic spaces we can define any conservation laws with respect to $N$-adapted anholonomic frames; the invariants of such conservations laws are not constant with respect to coordinate frames) of anholonomic motion $J(u, \Pi, \psi)$, the bracket with $H$ vanishes, $\{H, J\}=0$, where the Poisson-Dirac brackets for functions of the covariant phase-space variables $(u, \Pi, \psi)$ is defined

$$
\begin{aligned}
\{F, G\}= & D_{\mu} F \frac{\partial G}{\partial \Pi_{\mu}}-\frac{\partial F}{\partial \Pi_{\mu}} D_{\mu} G-R_{\mu \nu} \frac{\partial F}{\partial \Pi_{\mu}} \frac{\partial G}{\partial \Pi_{\nu}}+i(-1)^{a_{F}} \frac{\partial F}{\partial \psi^{\mu}} \frac{\partial G}{\partial \psi_{\mu}} \\
= & D_{i} F \frac{\partial G}{\partial \Pi_{i}}-\frac{\partial F}{\partial \Pi_{i}} D_{i} G-R_{i j} \frac{\partial F}{\partial \Pi_{i}} \frac{\partial G}{\partial \Pi_{j}}+i(-1)^{a_{F}} \frac{\partial F}{\partial \psi^{i}} \frac{\partial G}{\partial \psi_{i}} \\
& +D_{a} F \frac{\partial G}{\partial \Pi_{a}}-\frac{\partial F}{\partial \Pi_{a}} D_{a} G-R_{a b} \frac{\partial F}{\partial \Pi_{a}} \frac{\partial G}{\partial \Pi_{b}}+i(-1)^{a_{F}} \frac{\partial F}{\partial \psi^{a}} \frac{\partial G}{\partial \psi_{a}} .
\end{aligned}
$$

In definition of $\{\cdots\}$ there are used the operators

$$
\begin{aligned}
& D_{\mu} F=\partial_{\mu}+\Gamma_{\mu \nu}^{\lambda} \Pi_{\lambda} \frac{\partial F}{\partial \Pi_{\nu}}-\Gamma_{\mu \nu}^{\lambda} \psi^{\nu} \frac{\partial F}{\partial \psi^{\lambda}}, \\
& R_{\mu \nu}=\frac{i}{2} \psi^{\rho} \psi^{\sigma} R_{\rho \sigma \mu \nu},
\end{aligned}
$$


where on anisotropic spaces $\Gamma_{\mu \nu}^{\lambda}$ is the canonical d-connection (19), $R_{\rho \sigma \mu \nu}$ is the curvature d-tensor (21) with components (22) and $a_{F}$ is the Grassmann parity of $F: a_{F}=(0,1)$ for $F=($ even, odd $)$.

\subsubsection{Anisotropic Killing equations}

Expanding $J(u, \Pi, \psi)$ in a power series on the canonical momentum, $J=\sum_{n=0}^{\infty} \frac{1}{n !} \times$ $J^{(n) \mu_{1} \ldots \mu_{n}}(u, \psi) \Pi_{\mu_{1}} \cdots \Pi_{\mu_{n}}$ we conclude that the bracket $\{H, J\}$ vanishes for arbitrary $\Pi_{\mu}$ if and only if the components of $J$ satisfy the generalized anisotropic Killing equations [23]:

$$
J_{\left(\mu_{1} \ldots \mu_{n} ; \mu_{n+1}\right)}^{(n)}+\frac{\partial J_{\left(\mu_{1} \ldots \mu_{n}\right.}^{(n)}}{\partial \psi^{\sigma}} \Gamma_{\left.\mu_{n+1}\right) \lambda}^{\sigma} \psi^{\lambda}=\frac{i}{2} \psi^{\rho} \psi^{\sigma} R_{\rho \sigma v\left(\mu_{n+1}\right.} J_{\left.\mu_{1} \ldots \mu_{n}\right)}^{(n+1) v},
$$

where the round brackets $(\cdots)$ denote full symmetrization over the indices enclosed and the covariant derivation ";" is defined by the canonical d-connection (19) and one should be emphasized that every Greek index split into horizontal and vertical groups like $\mu_{n}=\left(i_{n}, a_{n}\right)$ which results that this equation will contain both "pure" horizontal or vertical terms as well terms of "mixed" character, like $J_{i_{1} \ldots a_{n}}^{(n)}$.

The type of solutions of the generalized anisotropic Killing equations (49) is defined by two classes from the locally isotropic limit [24,25]: the first class of solutions are generic ones, which exists for any spinning particle model and the second class of solutions are non-generic ones, which depend on the specific background space and anisotropy considered.

\subsubsection{Generic solutions of Killing equations}

The proper-time translations and supersymmetry are generated by the Hamiltonian and supercharge

$$
Q_{0}=\Pi_{\mu} \psi^{\mu}=\Pi_{i} \psi^{i}+\Pi_{a} \psi^{a}
$$

and belong to the first class. There is also an additional "chiral" symmetry generated by the chiral charge

$$
\Gamma_{*}=\frac{i^{\left[\frac{d}{2}\right]}}{d !} \sqrt{g} \epsilon_{\mu_{1} \ldots \mu_{d}} \psi^{\mu_{1}} \ldots \psi^{\mu_{d}}
$$

and a dual supersymmetry with generator

$$
Q^{*}=i\left\{\Gamma_{*}, Q_{0}\right\}=\frac{i^{\left[\frac{d}{2}\right]}}{(d-1) !} \sqrt{g} \epsilon_{\mu_{1} \ldots \mu_{d}} \Pi^{\mu_{1}} \psi^{\mu_{2}} \cdots \psi^{\mu_{d}},
$$

where $d$ is the dimension of spacetime.

\subsubsection{Non-generic solutions and Killing-Yano d-tensors}

The non-generic conserved quantities depend on the explicit form of the metric $g_{\mu \nu}(u)$ and, in our case, on $N$-connection structure. Following Ref. [25], generalizing the constructions for anisotropic spaces, we introduce the Killing-Yano d-tensors as objects generating non-generic $N$-distinguished supersymmetries. A d-tensor $f_{\mu_{1} \ldots \mu_{r}}$ is 
called Killing-Yano of valence $r$ if it is totally antisymmetric and satisfies the equation $f_{\mu_{1} \ldots \mu_{r-1}\left(\mu_{r} ; \lambda\right)}=0$.

The method of solution of the system of coupled differential equations (49) is similar to the method developed for locally isotropic spaces [9], that why here we present only the key results which have to be split on $\mathrm{h}$ - and v-indices if we need explicit formulas for holonomic-anholonomic components.

We start with a $\tilde{J}_{\mu_{1} \ldots \mu_{n}}^{(n)}$ solution of the homogeneous equation:

$$
\tilde{J}_{\left(\mu_{1} \ldots \mu_{n} ; \mu_{n+1}\right)}^{(n)}+\frac{\partial \tilde{J}_{\left(\mu_{1} \ldots \mu_{n}\right.}^{(n)}}{\partial \psi^{\sigma}} \Gamma_{\left.\mu_{n+1}\right) \lambda}^{\sigma} \psi^{\lambda}=0
$$

This solution is introduced in the r.h.s. of (49) for $J_{\mu_{1} \ldots \mu_{n-1}}^{(n-1)}$ and the iteration is carried on to $n=0$.

For the bosonic case the first equation shows that $J_{0}$ is a trivial constant, the next one is the equation for the Killing d-vectors and so on. In general, the homogeneous equation for a given $n$ defines a Killing d-tensor $J_{\mu_{1} \ldots \mu_{n}}^{(n)}$ for which $J_{\mu_{1} \ldots \mu_{n}}^{(n)} \Pi^{\mu_{1}} \ldots \Pi^{\mu_{n}}$ is a first integral of the geodesic equation [26]. This does not hold for the spinning particles.

Let us consider the case $n=0$, when $\tilde{J}^{(0)}=\frac{i}{4} f_{\mu \nu} \psi^{\mu} \psi^{\nu}$ is a solution if $f_{\mu \nu}$ is a Killing-Yano d-tensor d-covariantly constant, i.e., $\tilde{J}^{(0)}$ is a separately conserved quantity. For $n=1$, the natural solution is: $\tilde{J}_{\mu}^{(1)}=R_{\mu} f_{\lambda \sigma} \psi^{\lambda} \psi^{\sigma}$, where $R_{\mu}$ is a Killing d-vector $\left(R_{(\mu ; \nu)}=0\right)$ and $f_{\lambda \sigma}$ is a Killing-Yano d-tensor d-covariantly constant. Introducing this solution in the r.h.s. of Eq. (49) with $n=0$, we get $J^{(0)}=\frac{i}{2} R_{[\mu ; \nu]} f_{\lambda \sigma} \psi^{\mu} \psi^{\nu} \psi^{\lambda} \psi^{\sigma}$, where the square bracket denotes the antisymmetrization with norm one.

We define a new integral of anholonomic motion which is peculiar to the spinning case and has its analogous in the locally isotropic limit:

$$
J=f_{\mu \nu} \psi^{\mu} \psi^{\nu}\left(R_{\lambda} \Pi^{\lambda}+\frac{i}{2} R_{[\lambda ; \sigma]} \psi^{\lambda} \psi^{\sigma}\right)
$$

We can generate another $\psi$-dependent solution of the $n=1$ by starting from a KillingYano d-tensor with $r$ indices, $\tilde{J}_{\mu_{1}}^{(1)}=f_{\mu_{1} \mu_{2} \ldots \mu_{r}} \psi^{\mu_{2}} \cdots \psi^{\mu_{r}}$, or, following the above prescription, we express

$$
J^{(0)}=\frac{i}{r+1}(-1)^{r+1} f_{\left[\mu_{1} \ldots \mu_{r} ; \mu_{r+1}\right]} \psi^{\mu_{1}} \cdots \psi^{\mu_{r+1}}
$$

stating that the integral of motion corresponding to these solutions of the Killing equations is:

$$
Q_{f}=f_{\mu_{1} \ldots \mu_{r}} \Pi^{\mu_{1}} \psi^{\mu_{2}} \cdots \psi^{\mu_{r}}+\frac{i}{r+1}(-1)^{r+1} f_{\left[\mu_{1} \ldots \mu_{r} ; \mu_{r+1}\right]} \psi^{\mu_{1}} \cdots \psi^{\mu_{r+1}} .
$$

We conclude that the existence of a Killing-Yano d-tensor with $r$ indices is equivalent to the existence of a supersymmetry for the spinning space with supercharge $Q_{f}$ which anticommutes with $Q_{0}$, such constructions are anholonomic and distinguished by the $N$-connection structure. 


\section{Anisotropic Taub-NUT spinning space}

There are four Killing-Yano tensors in the usual, locally isotropic, Taub-NUT geometry [27] which for anisotropic spaces are transformed into corresponding d-tensors for anisotropic Taub-NUT spaces,

$$
f_{i}=8 m(r, \theta, s) \delta \varsigma \wedge \delta x_{i}-\epsilon_{i j k}\left(1+\frac{4 m(r, \theta, s)}{r}\right) \delta x_{j} \wedge \delta x_{k},
$$

which are d-covariantly constant and the fourth Killing-Yano d-tensor is

$$
f_{Y}=8 m(r, \theta, s) \delta \varsigma \wedge d r+4 r(r+2 m(r, \theta, s))\left(1+\frac{r}{4 m(r, \theta, s)}\right) \sin \theta d \theta \wedge \delta \varphi
$$

having only one non-vanishing component of the field strength $f_{Y r \theta ; \varphi}=2(1+$ $r / 4 m(r, \theta, s)) r \sin \theta$, where $\delta \varsigma=d \varsigma+\cos \theta d \theta$, and the values $\delta x_{j}$ are $N$-elongated for the v-components.

The corresponding supercharges constructed from the Killing-Yano d-tensors are $Q_{i}$ and $Q_{Y}$. The supercharges $Q_{i}$ together with $Q_{0}$ from (50) realize the $N=4$ supersymmetry algebra [28], in our case distinguished by the $N$-connection structure [16]:

$$
\left\{Q_{A}, Q_{B}\right\}=-2 i \delta_{A B} H, \quad A, B=0, \ldots, 3,
$$

making manifest the link between the existence of the Killing-Yano d-tensors and the hyper-Kähler d-geometry of the anisotropic Taub-NUT manifold. Such distinguished manifolds and geometries are constructed as the usual ones but with respect to $N$-connection decompositions on holonomic-anholonomic variables.

Starting with these results from the bosonic sector of the Taub-NUT space one can proceed with the spin contributions. The first generalized Killing equation (49) shows that with each Killing vector $R_{A}^{\mu}$ there is an associated Killing scalar $B_{A}$. The expression for the Killing scalar is taken as in Ref. [28]:

$$
B_{A}=\frac{i}{2} R_{A[\mu ; \nu]} \psi^{\mu} \psi^{v}
$$

with that modification that we use a d-covariant derivation which gives that the total angular momentum and "relative electric charge" become in the anisotropic spinning case

$$
\vec{J}=\vec{B}+\vec{j}, \quad J_{0}=B_{0}+q,
$$

where $\vec{J}=\left(J_{1}, J_{2}, J_{3}\right)$ and $\vec{B}=\left(B_{1}, B_{2}, B_{3}\right)$ are given with respect to anholonomic bases. These integrals of motion are superinvariant: $\left\{J_{A}, Q_{0}\right\}=0, A=0, \ldots, 3$.

We can introduce a Lie algebra with anholonomic constraints defined by the Killing d-vectors and realized by the integral of motion through the Poisson-Dirac brackets.

Now, introducing the Killing d-tensors $\vec{K}_{\mu \nu}$ into the generalized anisotropic Killing equation (49) we obtain that the corresponding Killing d-vectors $\vec{R}_{\mu}$ have a spin dependent part $\vec{S}_{\mu}$ as in the locally isotropic case [9], $\vec{R}_{\mu}=\vec{R}_{\mu}+\vec{S}_{\mu}$, where $\vec{R}_{\mu}$ are the standard Killing d-vectors. The $\psi$-dependent parts of the Killing d-vectors $\vec{S}_{\mu}$ contributes to the 
Runge-Lenz d-vector for the anisotropic spinning space

$$
\vec{K}=\frac{1}{2} \vec{K}_{\mu \nu} \cdot \frac{d u^{\mu}}{d \tau} \frac{d u^{\nu}}{d \tau}+\vec{S}_{\mu} \cdot \frac{d u^{\mu}}{d \tau},
$$

or in terms of the supercharges $Q_{\underline{i}}$ and $Q_{Y}$,

$$
\vec{K}_{i}=i\left\{Q_{Y}, Q_{\underline{i}}\right\}, \quad \underline{i}=1,2,3,
$$

we are using underlined Latin indices like $\underline{i}, \underline{j}, \ldots$ enumerating the number of supercharges, in order to distinguish them from the h-indices $i, j, \ldots$ used for holonomic variables on anisotropic spacetime.

The non-vanishing Poisson brackets are (after some algebra):

$$
\begin{aligned}
& \left\{J_{\underline{i}}, J_{\underline{j}}\right\}=\epsilon_{\underline{i} \underline{j} \underline{k}} J_{\underline{k}}, \quad\left\{J_{\underline{i}}, K_{\underline{j}}\right\}=\epsilon_{\underline{i} \underline{j} \underline{k}} K_{\underline{k}}, \\
& \left\{K_{\underline{i}}, K_{\underline{j}}\right\}=\left(\frac{J_{0}^{2}}{16 m^{2}}-2 E\right) \epsilon_{\underline{i} \underline{j} \underline{k}} J_{\underline{k}},
\end{aligned}
$$

where the values have anisotropic dependencies. Following the fact of existence of the Killing-Yano covariantly constants d-tensors $f_{\underline{i}} \mu \nu$, we can define three integrals of motion: $S_{i}=\frac{i}{4} f_{\underline{i} \mu \nu} \psi^{\mu} \psi^{\nu}, \underline{i}=1,2,3$, which realize an $S O(3)$ Lie algebra similar to that of the angular momentum: $\left\{S_{\underline{i}}, S_{\underline{j}}\right\}=\epsilon_{\underline{i} \underline{j} \underline{k}} S_{\underline{k}}$.

These components of the spin are separately conserved, do not depend on the frame of reference, holonomic or anholonomic, and can be combined with the angular momentum $\vec{J}$ in order to get a new improved form of the angular momentum $I_{\underline{i}}=J_{\underline{i}}-S_{\underline{i}}$ with the property that one preserves the algebra $\left\{I_{\underline{i}}, I_{\underline{j}}\right\}=\epsilon_{\underline{i}} \underline{j} \underline{\underline{k}} I_{\underline{k}}$ and that it commutes with the $S O(3)$ algebra generated by the spin $S_{\underline{i}},\left\{I_{\underline{i}}, S_{\underline{j}}\right\}=0$.

Let us consider the Dirac brackets of $\bar{S}_{i}$ with supercharges $\left\{S_{\underline{i}}, Q_{0}\right\}=-Q_{\underline{i}} / 2$; $\left\{S_{\underline{i}}, Q_{\underline{j}}\right\}=\frac{1}{2}\left(\delta_{\underline{i}} \underline{j} Q_{0}+\epsilon_{\underline{i} \underline{j}} O_{\underline{k}}\right)$. We can combine the above presented two $S O(3)$ algebras to obtain the generators of a conserved $S O(4)$ symmetry among the constants of motion, a standard basis for which is spanned by $M_{\underline{i}}^{ \pm}=I_{\underline{i}} \pm S_{\underline{i}}$.

There are also possible the combinations [9]:

$$
\begin{aligned}
\tilde{J}_{A \underline{j} \mu}^{(1)} & =R_{A \mu} f_{\underline{j} \lambda \sigma} \psi^{\lambda} \psi^{\sigma}, \quad A=0, \ldots, 3, \\
J_{A \underline{j}} & =f_{\underline{j}_{\lambda} \sigma} \psi^{\lambda} \psi^{\sigma}\left(R_{A \mu} \Pi^{\mu}+\frac{i}{2} R_{A[\alpha ; \beta]} \psi^{\alpha} \psi^{\beta}\right) \\
& =-4 i S_{\underline{j}} J_{A}, \quad A=0, \ldots, 3, \underline{j}=1,2,3 .
\end{aligned}
$$

So, there are a sort of Runge-Lenz d-vectors involving only Grassmann components:

$$
L_{\underline{i}}=\frac{1}{m} \epsilon_{\underline{i}} \underline{\underline{k}} \underline{S_{j}} J_{\underline{k}}, \quad \underline{i}, \underline{j}, \underline{k}=1,2,3,
$$

with the commutation relations:

$$
\left\{L_{\underline{i}}, J_{\underline{j}}\right\}=\epsilon_{\underline{i}} \underline{j} \underline{k} J_{\underline{k}}, \quad\left\{L_{\underline{i}}, L_{\underline{j}}\right\}=\left(\vec{S} \vec{J}-\vec{S}^{2}\right) \frac{1}{m^{2}} \epsilon_{\underline{i}} \underline{j} \underline{k} J_{\underline{k}} .
$$


Finally, we note the following Dirac brackets of $L_{\underline{i}}$ with supercharges:

$$
\begin{aligned}
& \left\{L_{\underline{i}}, Q_{0}\right\}=-\frac{1}{2 m} \epsilon_{\underline{i}} \underline{j} \underline{k} J_{\underline{k}} Q_{\underline{j}}, \\
& \left\{L_{\underline{i}}, Q_{\underline{j}}\right\}=\frac{1}{2 m}\left(\epsilon_{\underline{i}} \underline{j} \underline{k} Q_{0} J_{\underline{k}}-\delta_{\underline{i}} \underline{j} Q_{\underline{k}} M_{\underline{k}}^{-}+Q_{\underline{i}} M_{\underline{j}}^{-}\right),
\end{aligned}
$$

and emphasize that the presented algebraic relations hold true for anisotropic (as on some parameters) dependencies of the constant $m=m(r, \theta, s)$ because we are working with respect to anholonomic frames locally adapted to the $N$-connection structure.

\section{Concluding remarks}

In this paper we have extended the method of construction of new exact solutions, with generic local anisotropy, of the Einstein equations by using anholonomic frames with associated nonlinear connection structure (the method was proposed and developed in Refs. [3,15-17]) in order to generate vacuum metrics defining locally anisotropic Taub-NUT instanton and Kaluza-Klein monopoles. Such metrics are off-diagonal with respect to usual coordinate bases and reflect possible interactions of gravitational fields with gauge fields, induced from higher-dimensional gravity, in a new fashion when the constants interactions could run on the 5 th dimension and/or polarized to some anisotropic configurations on angular coordinates.

The first key result of this paper is the proof that the introduced ansatz for the metric and anholonomy coefficients substantially simplifies the structure of resulting vacuum gravitational field equations, the variables being separated and the system of nonlinear partial equations admitting exact solutions. In consequence, a straightforward definition of new classes of anisotropic Taub-NUT metrics with the effective constant $m$ varying on the 5th coordinate, as well with anisotropies and elliptic polarizations on angular coordinates, was possible. There were emphasized classes of anisotropic Taub-NUT wormhole solutions which can be generalized to various type of rotation hypersurface backgrounds and deformations.

The second key result is connected with the definition of integrals of motion of scalar and spinning particles in curved spacetimes provided with anholonomic frame structure. We proved that the symmetries of such generalized anisotropic Taub-NUT spaces are connected with anholonomic Killing vectors and tensors which are subjected to some anholonomic conservation laws. The problem of generation of non-generic anisotropic supersymmetries was solved by introducing Killing-Yano tensors adapted to the anholonomic spacetime structure.

Finally, we note that the results of this paper were extended for 3D solitonic configurations [5].

\section{Acknowledgements}

The authors thank M. Visinescu for hospitality, substantial support and discussion of results. S.V. is grateful to D. Singleton, E. Gaburov and D. Gontsa for collaboration 
and discussing of results. He also thanks V. Manu for support and help. The work is supported both by "The 2000-2001 California State University Legislative Award" and a NATO/Portugal fellowship for the Instituto Superior Tecnico, Lisabon.

\section{References}

[1] A. Salam, J. Strathdee, Ann. Phys. (N.Y.) 141 (1982) 316;

R. Percacci, S. Randjbar-Daemi, J. Math. Phys. 24 (1983) 807-814.

[2] A. Chodos, S. Detweiler, Gen. Relativ. Gravit. 14 (1982) 879;

G. Clément, Gen. Relativ. Gravit. 16 (1984) 131;

V.D. Dzhunushaliev, Izv. Vuzov, ser. Fizika 78 (6) (1993) (in Russian);

V.D. Dzhunushaliev, Gravit. Cosmol. 3 (1997) 240;

V.D. Dzhunushaliev, Gen. Relativ. Gravit. 30 (1998) 583;

V.D. Dzhunushaliev, Mod. Phys. Lett. A 13 (1998) 2179;

V.D. Dzhunushaliev, D. Singleton, Class. Quantum Grav. 16 (1999) 973.

[3] S. Vacaru, gr-qc/0001020;

S. Vacaru, JHEP 0104 (2001) 009;

S. Vacaru, Ann. Phys. (N.Y.) 290 (2001) 83.

[4] S. Vacaru, D. Singleton, V. Botan, D. Dotenco, Phys. Lett. B 519 (2001) 249;

S. Vacaru, D. Singleton, Ellipsoidal, cylindrical, bipolar and toroidal wormholes in 5D gravity, hep-th/ 0110272 .

[5] S. Vacaru, F.C. Popa, Class. Quantum Grav. 18 (2001) 4921.

[6] S. Hawking, Phys. Lett. A 60 (1977) 81.

[7] N.S. Manton, Phys. Lett. B 110 (1985) 54;

N.S. Manton, Phys. Lett. B 154 (1985) 397;

N.S. Manton, Phys. Lett. B 157 (1985) 475.

[8] M.F. Atiyah, N. Hitchin, Phys. Lett. A 107 (1985) 21.

[9] M. Visinescu, Class. Quantum Grav. 11 (1994) 1867;

M. Visinescu, Phys. Lett. B 339 (1994) 28;

D. Vaman, M. Visinescu, Phys. Rev. D 54 (1996) 1398;

D. Vaman, M. Visinescu, Phys. Rev. D 57 (1997) 3790;

D. Vaman, M. Visinescu, Fortschr. Phys. 47 (1999) 493.

[10] S. Vacaru, Off-diagonal 5D metrics and mass hierarchies with anisotropies and running of constants, hep-ph/ 0106268;

S. Vacaru, A new method of constructing black hole solutions in Einstein and 5D dimension gravity, hep-th/ 0110250.

[11] N. Arkani-Hamed, S. Dimopoulos, G. Dvali, Phys. Lett. B 429 (1998) 263, hep-th/9803315;

N. Arkani-Hamed, S. Dimopoulos, G. Dvali, Phys. Rev. D 59 (1999) 086004, hep-th/9807344;

I. Antoniadis, N. Arkani-Hamed, S. Dimopoulos, G. Dvali, Phys. Lett. B 436 (1998) 257, hep-th/9804398;

N. Arkani-Hamed, S. Dimopoulos, J. March-Russel, Phys. Rev. D 63 (2001) 064020, hep-th/9809124;

L. Randall, R. Sundrum, Phys. Rev. Lett. 83 (1999) 3370, hep-ph/9905221;

L. Randall, R. Sundrum, Phys. Rev. Lett. 83 (1999) 4690, hep-th/9906064.

[12] R.D. Sorkin, Phys. Rev. Lett. 51 (1983) 87.

[13] V.D. Dzhunushaliev, D. Singleton, Phys. Rev. D 59 (1999) 0664018.

[14] D.J. Gross, M.J. Perry, Nucl. Phys. B 226 (1983) 29.

[15] S. Vacaru, Phys. Lett. B 498 (2001) 74;

S. Vacaru, Gauge gravity and conservation laws in higher order anisotropic spaces, hep-th/9810229;

S. Vacaru, H. Dehnen, Locally anisotropic structures and nonlinear connections in Einstein and gauge gravity, gr-qc/0009039;

S. Vacaru, S. Ostaf, Buletinul Academiei de Stiinte a Republici Moldova, Fizica si Tehnica, Izvestia Akademii Nauk Respubliki Moldova 3 (12) (1993) 4;

S. Vacaru, Yu. Goncharenko, Int. J. Theor. Phys. 34 (1995) 1955. 
[16] S. Vacaru, Ann. Phys. (N.Y.) 256 (1997) 39;

S. Vacaru, Nucl. Phys. B 434 (1997) 590.

[17] S. Vacaru, J. Math. Phys. 37 (1996) 508;

S. Vacaru, JHEP 09 (1998) 011;

S. Vacaru, P. Stavrinos, Spinors and Space-Time Anisotropy, Athens Univ. Press, 2002.

[18] E. Cartan, Les Espaces de Finsler, Herman, Paris, 1934.

[19] W. Barthel, J. Reine Angew. Math. 212 (1963) 120.

[20] R. Miron, M. Anastasiei, The Geometry of Lagrange Spaces: Theory and Applications, Kluwer, 1994.

[21] A. Comtet, P.H. Horvathy, Phys. Lett. B 349 (1995) 49.

[22] R.H. Rietjik, J.W. van Holten, Nucl. Phys. B 472 (1996) 427;

R.H. Rietjik, J.W. van Holten, hep-th/9511166.

[23] See, e.g., R.H. Rietdijk, Applications of supersymmetric quantum mechanics, Ph.D. Thesis, Univ. Amsterdam, 1992, and references therein.

[24] R.H. Rietdijk, J.W. van Holten, Class. Quantum Grav. 7 (1990) 247.

[25] G.W. Gibbons, R.H. Rietdijk, J.W. van Holten, Nucl. Phys. B 404 (1993) 42.

[26] W. Dietz, R. Rüdinger, Proc. R. Soc. London A 375 (1981) 361.

[27] G.W. Gibbons, P.J. Ruback, Phys. Lett. B 188 (1987) 226;

G.W. Gibbons, P.J. Ruback, Commun. Math. Phys. 115 (1988) 267.

[28] J.W. van Holten, Phys. Lett. B 342 (1995) 47. 